\section{Carência de atenção à saúde ocular no setor público: um estudo de base populacional}

\author{
Shortage of ocular health care in the public \\ system: a population-based study
}

\author{
1 Universidade Federal de \\ Pelotas, Pelotas, Brasil. \\ Correspondência \\ V. D. Castagno \\ Universidade Federal \\ de Pelotas. \\ Rua Marechal Deodoro 1160, \\ Pelotas, RS \\ 96020-220, Brasil. \\ vicastagno@viacabo.com.br
}

\section{Abstract}

This cross-sectional population-based study investigated the prevalence of eye care services utilization and the association with socioeconomic and demographic factors, need for health care, and type of service payment. The study evaluated 2,960 adults aged 20 and older. In the previous 5 years, $46 \%$ of the sample and $30 \%$ of those aged 50 and older had not visited an eye care service. Among the persons who used a service, 18\% went to an optical store and only $17 \%$ used the public health system. The main reason for using eye care services was poor vision (69.5\%). Lack of money (29\%) and time (24.6\%) were the most frequently cited reasons for non-utilization. Age, education, and economic status were directly associated with the outcome. Female gender, cataract, glaucoma, prescription eyewear, and private appointments were also associated with the outcome. It is necessary to increase the public health system's role, integrating ocular health with all levels of health care, expanding participation by other health professionals, and intensifying screening and prevention of eye problems.

Ophtalmological Services; Eye Health; Public Sector
Victor Delpizzo Castagno 1 Anaclaudia Gastal Fassa 1 Marcelo Cozzensa da Silva ${ }^{1}$ Maria Laura Vidal Carret 1

\section{Introdução}

A baixa acuidade visual é um problema de alta prevalência, que freqüentemente tem impacto negativo sobre a qualidade de vida, implicando, inclusive, restrições ocupacionais, econômicas e sociais. No mundo, 314 milhões de pessoas apresentam dificuldade visual ${ }^{1}$. No Brasil, estima-se que, da população geral, $14,5 \%$ apresentam alguma deficiência e, destes, $48,1 \%$ são deficientes visuais ${ }^{2}$, ou seja, quase 12 milhões de pessoas. Porém, segundo a Organização Mundial da Saúde (OMS), 75\% de toda cegueira são tratáveis e/ ou evitáveis 1 .

Não existem evidências que dêem suporte às recomendações sobre a periodicidade de realização de consultas para os olhos, em cada faixa etária 3. De acordo com Bylsma et al. 4, o grupo VISION 2020: The Right to Sight, da Austrália, propôs um intervalo de utilização de serviços de saúde ocular de cinco anos para a população geral e de dois anos para aqueles que tenham diabetes melito ou história familiar de doença ocular. Já a Academia Americana de Optometria (AOA) recomenda consultas a cada dois anos para pessoas assintomáticas entre 18 e 60 anos de idade e anualmente para aquelas com 61 anos ou mais 5 .

Os estudos sobre prevalência de utilização de serviço de saúde ocular nos últimos cinco anos apresentaram resultados muito variáveis, sendo $56 \%$ para aqueles abaixo dos 40 anos 6 e entre 
$69 \%$ e $89 \%$ para os com 40 anos ou mais $4,6,7,8$. Estudo finlandês, avaliando população com baixa visão, encontrou uma prevalência de $58 \%$ de utilização de serviço de saúde ocular nos últimos cinco anos 9 . Idade e escolaridade estiveram diretamente associadas à utilização de serviços de saúde ocular. Ser mulher e apresentar mais necessidades em saúde ocular, como, por exemplo, glaucoma, diabetes melito e uso de correção (óculos ou lentes de contato) também estiveram positivamente associadas com o desfecho $4,6,7,8,10,11,12,13,14$.

A demanda por serviços de saúde ocular está concentrada na avaliação de problemas refrativos. Entretanto, o diagnóstico precoce e o tratamento das morbidades oculares crônicas, como a catarata, o glaucoma e a retinopatia diabética, são importantes demandas potenciais ${ }^{1}$. A oferta de serviços de saúde ocular no Brasil é limitada, especialmente no setor público, e centrada no oftalmologista. No Brasil, em 2001, para cada 100 mil habitantes, existiam seis oftalmologistas, concentrados em 677 dos 5.507 municípios brasileiros, fato que aponta para a necessidade de aumentar o número de profissionais da saúde envolvidos com o cuidado de saúde ocular nos diferentes níveis de atenção 15 .

Apesar da grande freqüência dos problemas de saúde ocular e de seu impacto na qualidade de vida 1, bem como dos indicativos de restrição na oferta de serviços, não foram encontrados estudos brasileiros de base populacional sobre a utilização de serviço de saúde ocular. Assim, esta pesquisa objetiva investigar a prevalência de utilização de serviços de saúde ocular, descrever o principal motivo da última consulta e o principal motivo para ter deixado de consultar nos últimos cinco anos. Também será avaliada a associação dos fatores demográficos, sócio-econômicos, de necessidades em saúde e de acesso a serviços de saúde ocular com o desfecho.

\section{Metodologia}

Realizou-se um estudo transversal de base populacional em Pelotas, cidade de porte médio do Sul do Brasil, com adultos de 20 anos ou mais, residentes na zona urbana, no período de outubro de 2007 a janeiro de 2008. Este estudo fez parte de um consórcio de pesquisas dos mestrandos do Curso de Pós-graduação em Epidemiologia da Universidade Federal de Pelotas (UFPEL), tendo sido investigados diferentes tópicos em uma mesma população de estudo.

A cidade onde o estudo foi realizado conta, no Sistema Único de Saúde (SUS), com 51 Unidades Básicas de Saúde (UBS) que dispõem, semanalmente, de até cinco encaminhamentos de pacientes para consulta com especialistas, incluindo a consulta com o médico oftalmologista. As consultas oftalmológicas são, então, realizadas em locais credenciados pelo SUS por nove oftalmologistas que atendem adultos e crianças (3 oftalmologistas/100 mil habitantes). No ano de 2007, na cidade estudada, foram realizadas 8.314 consultas oftalmológicas no setor público 16 .

A amostra estudada $(n=2.960)$ permitiu estimar a prevalência de utilização de serviços de saúde ocular nos últimos cinco anos, com um erro de dois pontos percentuais. A avaliação da associação entre toxoplasmose e utilização de serviço de saúde ocular foi a que necessitou de maior tamanho de amostra. Para essa associação, que apresentou relação exposto/não-exposto de 49:1 e prevalência de utilização de serviço de saúde ocular de $53 \%$ nos não expostos, obteve-se um poder estatístico de $80 \%$ para detectar razões de prevalência de 1,4 ou mais com nível de $95 \%$ de confiança.

A seleção da amostra foi realizada em dois estágios. No primeiro foram sorteados, de forma sistemática, levando em conta a renda média do chefe da família, 126 setores censitários. Essa amostragem foi proporcional ao tamanho do setor. No segundo, foram amostrados 11 domicílios por setor. Para isso, em cada setor foi realizada a contagem do número de domicílios, dividindose, em seguida, por 11, para estabelecer um "pulo", de forma a incluir domicílios distribuídos em todo o setor. Em cada domicílio amostrado foram estudados todos os indivíduos com 20 anos ou mais.

As entrevistas foram realizadas por 30 entrevistadoras selecionadas especificamente para este estudo, submetidas a um treinamento de 40 horas, com conteúdos teórico-práticos e entrevistas supervisionadas. Cada mestrando fez a supervisão de cerca de três entrevistadoras em oito setores censitários. As informações foram coletadas através de questionário padronizado, pré-codificado, constituído principalmente por questões fechadas. A codificação foi realizada pelas entrevistadoras logo após a entrevista e revisada pelos supervisores. Estes repetiram parte da entrevista em $10 \%$ da amostra para controle de qualidade, obtendo um kappa para utilização de correção de 0,8 .

As variáveis demográficas estudadas foram sexo (masculino ou feminino), idade (em anos completos) e cor da pele auto-referida (preta, parda, branca e outras). Com relação às variáveis sócio-econômicas, coletaram-se escolaridade, em anos completos de estudo, nível econômico, segundo a Associação Brasileira de Empresas de Pesquisa (ABEP) 17, e renda familiar mensal. Com 
relação às necessidades em saúde, caracterizaram-se as patologias oculares e as patologias sistêmicas com repercussão ocular mais freqüentes, avaliando o uso de algum tipo de correção ótica e o conhecimento prévio do entrevistado sobre diagnóstico médico de diabetes melito ou "açúcar no sangue", hipertensão arterial sistêmica ou "pressão alta", toxoplasmose ou "doença do gato", catarata e glaucoma ou "pressão alta nos olhos". O acesso a serviços de saúde ocular foi avaliado conforme a forma de financiamento da última consulta para os olhos, com base na informação sobre o local da última consulta. A forma de financiamento foi categorizada em público e privado/plano de saúde. De forma descritiva, o acesso a serviços de saúde ocular foi também avaliado pelo tempo que levou para consultar e pelo motivo para não ter consultado nos últimos cinco anos quando sentiu necessidade.

Para operacionalizar o desfecho, identificouse há quanto tempo o entrevistado realizou a última consulta para os olhos, seja com oftalmologista, seja com outro profissional da saúde - excetuando exames iniciais ou de renovação para Carteira Nacional de Habilitação $(\mathrm{CNH})$-, categorizando em nunca consultou; consultou há menos de 1 ano; entre 1 e 5 anos; há mais de 5 anos; consultou, mas não lembra quando. Para este artigo, dicotomizou-se o desfecho em consultou há menos de cinco anos e consultou há mais de cinco anos ou nunca consultou. Este ponto de corte foi escolhido pela possibilidade de avaliar a consistência em relação a outros estudos 4,6,7,8,9 e examinar a adequação às recomendações do grupo australiano ${ }^{4}$. Além disso, avaliou-se o principal motivo da última consulta para os olhos.

O banco de dados foi construído no programa Epi Info (Centers for Disease Control and Prevention, Atlanta, Estados Unidos). As informações foram duplamente digitadas, com checagem automática de consistência e amplitude das variáveis. A análise dos dados foi realizada no programa Stata, versão 9.0 (Stata Corp., College Station, Estados Unidos). A análise bruta avaliou as associações por meio dos testes de qui-quadrado para heterogeneidade e qui-quadrado para tendência linear.

A análise ajustada foi realizada através da regressão de Poisson com variância robusta e seleção para trás. O modelo de análise foi hierarquizado de acordo com o modelo conceitual do estudo. O modelo utilizado baseou-se na proposta Andersen 18, que tem sido usada em outros trabalhos sobre utilização de serviços de saúde ocular 6,8. Assim, os determinantes distais avaliados foram as variáveis sócio-econômicas e demográficas; os determinantes intermediários foram as variáveis de necessidade em saúde e o proximal foi a forma de financiamento da última consulta. As variáveis em cada nível foram ajustadas para as do mesmo nível e de níveis mais distais. Mantiveram-se, no modelo de análise, as variáveis que apresentaram $\mathrm{p} \leq 0,20$ para controle de fatores de confusão e consideraram-se estatisticamente significativos valores de $\mathrm{p}<0,05$.

Este estudo foi aprovado pelo Comitê de Ética em Pesquisa da Faculdade de Medicina da UFPEL e, como envolveu apenas coleta de dados mediante entrevista, foi considerado de baixo risco. Em adição, atendeu os requisitos da $D e$ claração de Helsinki - que normatiza a pesquisa em seres humanos -, dentre eles obtenção de consentimento informado por escrito, garantia do direito de não-participação e sigilo acerca das informações obtidas.

\section{Resultados}

Do total de pessoas elegíveis para o estudo, 2.960 foram entrevistadas $(6,1 \%$ de perdas e recusas). O coeficiente de correlação intraclasse e o efeito de delineamento para o desfecho foram de 0,079 e 2,81, respectivamente.

Entre os sujeitos do estudo, metade não consultou nos últimos cinco anos, e 30\% nunca consultaram para os olhos. Mesmo no grupo de mais de 50 anos, $30 \%$ não consultaram nos últimos cinco anos e $20 \%$ nunca consultaram. No grupo de menos de 50 anos, os percentuais foram, respectivamente, $55 \%$ e $37 \%$ (Figura 1 ).

Do total da amostra, $62,7 \%$ tinham entre $20 \mathrm{e}$ 49 anos; a média de idade foi de 44,5 anos (DP = 16,9). Dos entrevistados, 56,9\% eram mulheres e 75,6\% eram brancos. Quanto à escolaridade, $23,3 \%$ estudaram até quatro anos, e $30 \%$ daqueles com 50 anos ou mais tinham mais de oito anos de escolaridade. Os níveis econômicos mais baixos da ABEP (D+E) incluíram 16,1\% dos entrevistados, e $52,1 \%$ ganhavam até três salários mínimos (Tabela 1).

Ao avaliar as necessidades em saúde, 33,5\% dos sujeitos estudados e $54,4 \%$ dos com 50 anos ou mais relataram diagnóstico médico prévio de hipertensão arterial sistêmica. O relato de diabetes melito foi de 7,5\% para a totalidade da população estudada e de $14,9 \%$ para os com 50 anos ou mais. No que se refere à toxoplasmose, a prevalência total e naqueles com menos de 50 anos foi, respectivamente, $2,0 \%$ e 2,6\%. Entre aqueles que receberam diagnóstico médico para morbidades oculares específicas, $6,5 \%$ relataram catarata e $4,6 \%$, glaucoma. Nos sujeitos com 50 anos ou mais, a prevalência destas morbidades foi, respectivamente, 14,9\% e 7,9\%. O uso de ócu- 
Figura 1

Prevalência de consultas para os olhos conforme idade. Pelotas, Rio Grande do Sul, Brasil, 2008 ( $n=2.960)$

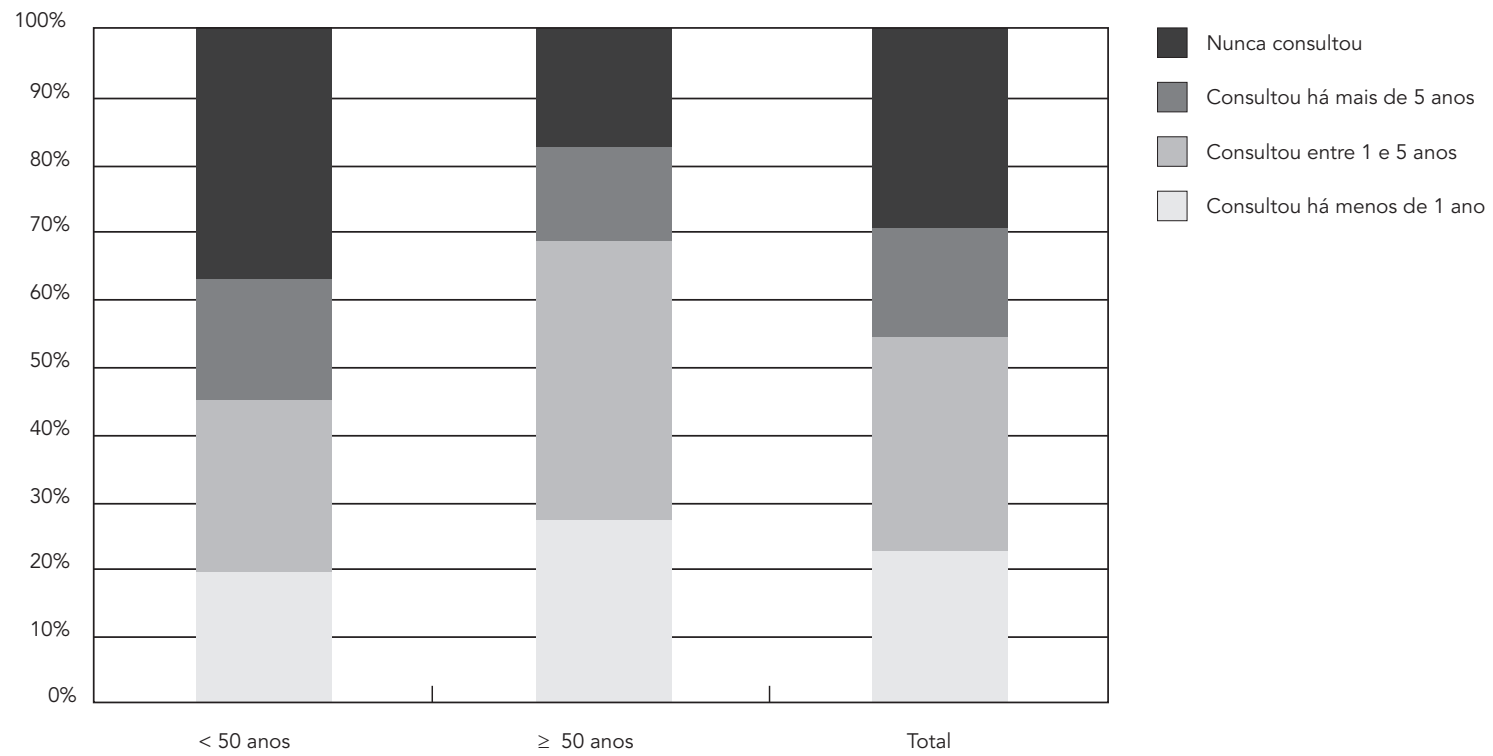

Tabela 1

Descrição da amostra segundo variáveis sócio-econômicas, demográficas, necessidades em saúde e acesso a serviços de saúde ocular conforme idade. Pelotas, Rio Grande do Sul, Brasil, 2008 ( $n=2.960)$.

\begin{tabular}{|c|c|c|c|c|c|c|}
\hline \multirow[t]{2}{*}{ Variável } & \multicolumn{2}{|c|}{$<50$ anos } & \multicolumn{2}{|c|}{$\geq \mathbf{5 0}$ anos } & \multicolumn{2}{|c|}{ Total } \\
\hline & $n$ & $\%$ & $n$ & $\%$ & $\mathrm{n}$ & $\%$ \\
\hline \multicolumn{7}{|l|}{ Sexo } \\
\hline Homem & 833 & 44,9 & 443 & 40,2 & 1.276 & 43,1 \\
\hline Mulher & 1.024 & 55,1 & 660 & 59,8 & 1.684 & 56,9 \\
\hline \multicolumn{7}{|c|}{ Idade (anos) } \\
\hline $20-39$ & 1.263 & 68,0 & - & - & 1.263 & 42,7 \\
\hline $40-49$ & 594 & 32,0 & - & - & 594 & 20,0 \\
\hline $50-64$ & - & - & 707 & 64,1 & 707 & 23,9 \\
\hline $65+$ & - & - & 396 & 35,9 & 396 & 13,4 \\
\hline \multicolumn{7}{|l|}{ Cor da pele } \\
\hline Preta & 277 & 14,9 & 124 & 11,3 & 401 & 13,6 \\
\hline Parda & 137 & 7,9 & 71 & 6,4 & 208 & 7,0 \\
\hline Branca & 1.367 & 73,7 & 867 & 78,7 & 2.234 & 75,6 \\
\hline Outras & 74 & 4,0 & 39 & 3,5 & 113 & 3,8 \\
\hline \multicolumn{7}{|c|}{ Escolaridade (anos de estudo) } \\
\hline $0-4$ & 255 & 13,7 & 435 & 39,5 & 690 & 23,3 \\
\hline $5-8$ & 592 & 31,9 & 340 & 30,8 & 932 & 31,5 \\
\hline $9-11$ & 607 & 32,7 & 154 & 13,9 & 761 & 25,7 \\
\hline $12+$ & 403 & 21,7 & 173 & 15,7 & 576 & 19,5 \\
\hline
\end{tabular}

(continua) 
Tabela 1 (continuação)

\begin{tabular}{|c|c|c|c|c|c|c|}
\hline \multirow[t]{2}{*}{ Variável } & \multicolumn{2}{|c|}{$<50$ anos } & \multicolumn{2}{|c|}{$\geq 50$ anos } & \multicolumn{2}{|c|}{ Total } \\
\hline & $\mathrm{n}$ & $\%$ & $\mathrm{n}$ & $\%$ & $\mathrm{n}$ & $\%$ \\
\hline \multicolumn{7}{|l|}{ Nível econômico (ABEP) } \\
\hline$A+B$ & 667 & 36,1 & 426 & 39,3 & 1.093 & 37,3 \\
\hline c & 905 & 49,0 & 460 & 42,4 & 1.365 & 46,6 \\
\hline$D+E$ & 274 & 14,8 & 198 & 18,3 & 472 & 16,1 \\
\hline \multicolumn{7}{|c|}{ Renda familiar (salários mínimos) } \\
\hline $0,0-1,0$ & 167 & 9,5 & 101 & 10,3 & 268 & 9,8 \\
\hline $1,1-3,0$ & 774 & 44,1 & 385 & 39,1 & 1.159 & 42,3 \\
\hline $3,1-6,0$ & 489 & 27,9 & 275 & 27,9 & 764 & 27,9 \\
\hline $6,1+$ & 323 & 18,4 & 223 & 22,5 & 546 & 20,0 \\
\hline \multicolumn{7}{|l|}{ Diabetes } \\
\hline Sim & 57 & 4,0 & 164 & 14,9 & 221 & 7,5 \\
\hline \multicolumn{7}{|l|}{ Hipertensão arterial sistêmica } \\
\hline Sim & 384 & 20,9 & 600 & 54,4 & 984 & 33,5 \\
\hline \multicolumn{7}{|l|}{ Toxoplasmose } \\
\hline Sim & 48 & 2,6 & 11 & 1,0 & 59 & 2,0 \\
\hline \multicolumn{7}{|l|}{ Catarata } \\
\hline $\operatorname{Sim}$ & 27 & 1,5 & 161 & 14,9 & 188 & 6,5 \\
\hline \multicolumn{7}{|l|}{ Glaucoma } \\
\hline Sim & 46 & 2,5 & 85 & 7,9 & 131 & 4,6 \\
\hline \multicolumn{7}{|l|}{ Uso de correção ocular } \\
\hline $\operatorname{Sim}$ & 598 & 32,2 & 878 & 79,6 & 1.476 & 49,9 \\
\hline \multicolumn{7}{|c|}{ Tempo que levou para consultar $(n=1.974)$} \\
\hline Até 24 horas & 590 & 54,0 & 461 & 52,2 & 1.051 & 53,2 \\
\hline De 2 dias até 1 semana & 350 & 32,1 & 290 & 32,8 & 640 & 32,4 \\
\hline Mais de 1 semana & 151 & 13,8 & 132 & 14,9 & 283 & 14,3 \\
\hline
\end{tabular}

ABEP: Associação Brasileira de Empresas de Pesquisa 17.

los ou lentes de contato foi referido por metade das pessoas, atingindo quase $80 \%$ na faixa etária de 50 anos ou mais (Tabela 1).

Metade dos entrevistados levou até 24 horas para realizar a consulta, contando a partir do momento que decidiu consultar (Tabela 1). A forma de financiamento da última consulta mostrou que somente $17 \%$ das pessoas que consultaram utilizaram o serviço público, sendo as demais consultas realizadas no setor privado por meio de convênio ou plano de saúde (42\%), consulta particular (23\%) e em óticas (18\%), não apresentando diferença conforme a faixa etária (Figura 2).

A dificuldade para enxergar foi o principal motivo de consulta em $70 \%$ dos casos, seguida de sintomas oculares agudos, como coceira, lacrimejamento, olho vermelho, sensação de areia nos olhos e pálpebras coladas, assim como os traumas oculares (16\%). Naqueles com 50 anos ou mais a dificuldade para enxergar compreendeu $80 \%$ dos motivos, e nos com menos de 50 anos os sintomas agudos atingiram $20 \%$. Dor de cabeça foi mais freqüente nas pessoas mais jovens $(9,8 \%)$, enquanto a consulta para acompanhamento de doenças sistêmicas ou oculares foi mais prevalente nas pessoas com 50 anos ou mais $(6,4 \%)$ (Tabela 2$)$.

Embora sentindo necessidade, $34 \%$ das pessoas não consultaram para os olhos nos últimos cinco anos. Os principais motivos para ter deixado de consultar foram: falta de dinheiro (29\%), falta de tempo (25\%) e descaso (19\%), sendo falta de tempo $(29,4 \%)$ mais importante para os menores de 50 anos e falta de dinheiro $(37,5 \%)$ o principal motivo para aqueles com 50 anos ou mais (Tabela 2).

A análise ajustada mostrou que mulheres consultaram $27 \%$ mais que os homens, (IC95\%: $1,19-1,36)$, e pessoas de cor branca consultaram 
Figura 2

Forma de financiamento da última consulta, entre os que consultaram para os olhos, de acordo com faixa etária. Pelotas, Rio Grande do Sul, Brasil, 2008 $(n=2.011) *$

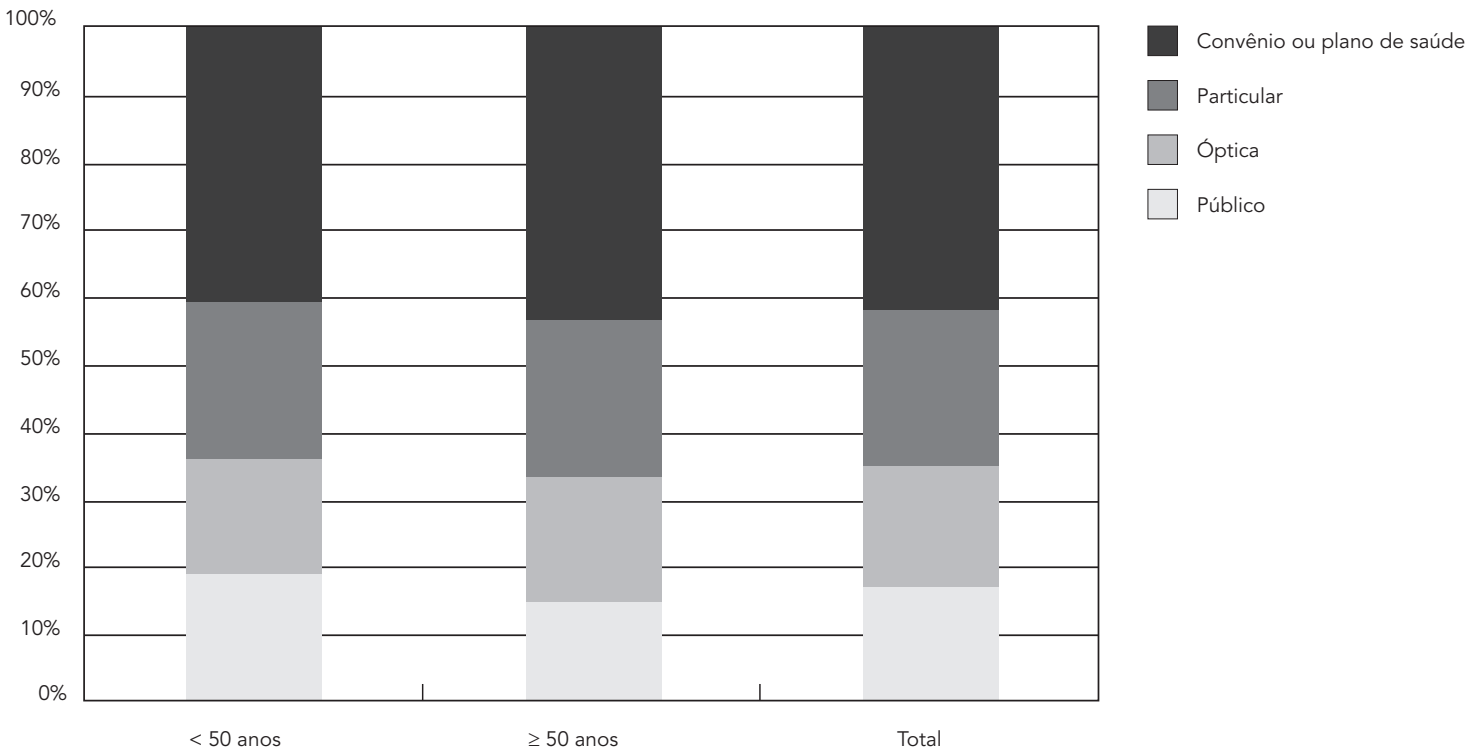

* Variável coletada somente para as pessoas que disseram ter consultado para os olhos alguma vez na vida (100 missings: 67 pessoas ignoravam onde haviam consultado e 33 consultaram em outras localidades sem informar se foi público ou privado).

Tabela 2

Características da utilização de serviços de saúde ocular de acordo com idade. Pelotas, Rio Grande do Sul, Brasil, 2008.

\begin{tabular}{|c|c|c|c|c|c|c|}
\hline \multirow[t]{2}{*}{ Variáveis } & \multicolumn{2}{|c|}{$<50$ anos } & \multicolumn{2}{|c|}{$\geq \mathbf{5 0}$ anos } & \multicolumn{2}{|c|}{ Total } \\
\hline & $\mathbf{n}$ & $\%$ & $\mathbf{n}$ & $\%$ & $\mathbf{n}$ & $\%$ \\
\hline \multicolumn{7}{|l|}{ Motivo da última consulta $(n=2.105)$} \\
\hline Não enxergar bem & 718 & 61,2 & 746 & 80,0 & 1.464 & 69,5 \\
\hline Sintomas agudos/traumas oculares & 240 & 20,5 & 103 & 11,1 & 343 & 16,3 \\
\hline Dor de cabeça & 115 & 9,8 & 9 & 1,0 & 124 & 5,9 \\
\hline Acompanhamento de doença ocular ou sistêmica & 31 & 2,6 & 60 & 6,4 & 91 & 4,3 \\
\hline Outros motivos & 69 & 5,9 & 14 & 1,5 & 83 & 3,9 \\
\hline \multicolumn{7}{|l|}{$\begin{array}{l}\text { Motivo de ter deixado de consultar alguma vez nos } \\
\text { últimos cinco anos }(n=1.004)\end{array}$} \\
\hline Falta de dinheiro & 156 & 24,2 & 135 & 37,5 & 291 & 29,0 \\
\hline Falta de tempo & 189 & 29,4 & 58 & 16,1 & 247 & 24,6 \\
\hline Descaso & 117 & 18,2 & 73 & 20,3 & 190 & 18,9 \\
\hline Dificuldade de marcar consulta & 85 & 13,2 & 38 & 10,6 & 123 & 12,2 \\
\hline Achar que o problema não era importante & 64 & 9,9 & 38 & 10,6 & 102 & 10,2 \\
\hline Outros motivos & 33 & 5,1 & 18 & 5,0 & 51 & 5,1 \\
\hline
\end{tabular}


$17 \%$ mais do que os negros (IC95\%: 1,02-1,33). Idade esteve diretamente associada com o desfecho: aqueles com 65 anos ou mais consultaram duas vezes mais do que os indivíduos com 20 a 39 anos. Quanto às variáveis sócio-econômicas, observou-se que escolaridade e nível econômico apresentaram efeitos independentes com relação ao desfecho (coeficiente de correlação de 0,50), permanecendo ambas diretamente associadas (Tabela 3). Assim, aqueles nas categorias de mais escolaridade e maior nível econômico consultaram cerca de $60 \%$ mais nos últimos cinco anos do que o grupo de base.

Dentre as variáveis de necessidade em saúde, aqueles que usavam correção ocular consultaram três vezes mais do que os que não usavam (IC95\%: 2,84-3,68), enquanto os que relatavam diagnóstico médico de catarata $(\mathrm{RP}=1,30) \mathrm{e}$ glaucoma consultaram 30\% (IC95\%: 1,18-1,42) e 22\% (IC95\%: 1,11-1,36) mais. Entretanto, mesmo sabendo ter estas morbidades, o percentual que não consultou nos últimos cinco anos foi de $15 \%$ e $20 \%$ respectivamente. Os relatos de diagnóstico de uma patologia sistêmica de potencial repercussão oftalmológica, como diabetes melito, hipertensão arterial sistêmica e toxoplasmose, não foram significativamente associados com o desfecho, e mais de $30 \%$ dos que relataram estas morbidades não consultaram nos últimos cinco anos (Tabela 3).

Em relação ao acesso aos serviços de saúde ocular, as pessoas que possuem planos de saúde ou pagam integralmente as consultas utilizaram $25 \%$ mais estes serviços nos últimos cinco anos, quando comparadas àquelas que consultaram serviços de públicos (IC95\%: 1,13-1,39) (Tabela3).

\section{Tabela 3}

Fatores associados a consulta para os olhos nos últimos cinco anos. Pelotas, Rio Grande do Sul, Brasil, 2008 ( $n=2.960$ ).

\begin{tabular}{|c|c|c|c|c|c|}
\hline Variáveis & $\%$ * & RP bruta (IC95\%) & Valor de $p$ & $\begin{array}{l}\text { RP ajustada } \\
(\text { IC95\%) ** }\end{array}$ & Valor de $p$ \\
\hline \multicolumn{6}{|l|}{ Primeiro nível } \\
\hline Sexo & & & $<0,001$ & & $<0,001$ \\
\hline Homens & 45,6 & 1,00 & & 1,00 & \\
\hline Mulheres & 58,6 & $1,28(1,20-1,38)$ & & $1,27(1,19-1,36)$ & \\
\hline Idade (anos) & & & $<0,001 * \star \star$ & & $<0,001 * \star \star$ \\
\hline $20-39$ & 41,6 & 1,00 & & 1,00 & \\
\hline $40-49$ & 48,5 & $1,16(1,05-1,29)$ & & $1,23(1,19-1,36)$ & \\
\hline $50-64$ & 65,9 & $1,58(1,44-1,74)$ & & $1,64(1,50-1,80)$ & \\
\hline $65+$ & 73,0 & $1,75(1,60-1,92)$ & & $2,02(1,82-2,25)$ & \\
\hline Cor da pele & & & $<0,001 \#$ & & 0,119 \# \\
\hline Preta & 39,9 & 1,00 & & 1,00 & \\
\hline Parda & 44,2 & $1,11(0,89-1,38)$ & & $1,08(0,87-1,36)$ & \\
\hline Branca & 56,6 & $1,49(1,23-1,64)$ & & $1,17(1,02-1,33)$ & \\
\hline Outras & 44,2 & $1,11(0,84-1,46)$ & & $1,09(0,85-1,39)$ & \\
\hline Escolaridade (anos de estudo) & & & $<0,001 \star \star \star$ & & 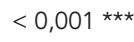 \\
\hline $0-4$ & 47,8 & 1,00 & & 1,00 & \\
\hline $5-8$ & 44,4 & $0,93(0,83-1,04)$ & & $1,04(0,92-1,16)$ & \\
\hline $9-11$ & 51,1 & $1,07(0,94-1,22)$ & & $1,25(1,09-1,44)$ & \\
\hline $12+$ & 75,5 & $1,58(1,42-1,75)$ & & $1,57(1,38-1,79)$ & \\
\hline Nível econômico (ABEP) & & & $<0,001 * \star \star$ & & $<0,001 * \star \star$ \\
\hline$D+E$ & 34,1 & 1,00 & & 1,00 & \\
\hline C & 46,7 & $1,37(1,18-1,59)$ & & $1,34(1,15-1,55)$ & \\
\hline$A+B$ & 68,8 & $2,02(1,74-2,34)$ & & $1,62(1,39-1,90)$ & \\
\hline
\end{tabular}

(continua) 


\begin{tabular}{|c|c|c|c|c|c|}
\hline Variáveis & $\%$ * & RP bruta (IC95\%) & Valor de $\mathrm{p}$ & $\begin{array}{l}\text { RP ajustada } \\
(\text { IC95\%) ** }\end{array}$ & Valor de $p$ \\
\hline \multicolumn{6}{|l|}{ Segundo nível } \\
\hline Diabete \#\# & & & $<0,001$ & & 0,116 \\
\hline Não & 52,0 & 1,0 & & 1,0 & \\
\hline Sim & 67,4 & $1,30(1,18-1,43)$ & & $1,07(0,98-1,17)$ & \\
\hline Hipertensão arterial sistêmica \#\# & & & $<0,001$ & & 0,399 \\
\hline Não & 49,8 & 1,0 & & 1,0 & \\
\hline Sim & 59,9 & $1,20(1,12-1,28)$ & & $0,97(0,91-1,04)$ & \\
\hline Catarata \#\# & & & $<0,001$ & & $<0,001$ \\
\hline Não & 51,8 & 1,0 & & 1,0 & \\
\hline Sim & 86,2 & $1,66(1,54-1,78)$ & & $1,30(1,18-1,42)$ & \\
\hline Glaucoma \#\# & & & $<0,001$ & & $<0,001$ \\
\hline Não & 52,8 & 1,0 & & 1,0 & \\
\hline Sim & 80,1 & $1,52(1,38-1,68)$ & & $1,22(1,11-1,36)$ & \\
\hline Toxoplasmose \#\# & & & 0,270 & & 0,137 \\
\hline Não & 53,1 & 1,0 & & 1,0 & \\
\hline Sim & 61,0 & $1,15(0,90-1,47)$ & & $1,13(0,96-1,33)$ & \\
\hline Uso de correção \#\# & & & $<0,001$ & & $<0,001$ \\
\hline Não & 23,2 & 1,0 & & 1,0 & \\
\hline Sim & 82,9 & $3,57(3,17-4,01)$ & & $3,23(2,84-3,68)$ & \\
\hline \multicolumn{6}{|l|}{ Terceiro nível \#\# } \\
\hline $\begin{array}{l}\text { Financiamento (última consulta) } \\
(\mathrm{n}=2.011)\end{array}$ & & & $<0,001$ & & $<0,001$ \\
\hline Público & 55,3 & 1,0 & & 1,0 & \\
\hline Privado/Convênio & 81,2 & $1,47(1,32-1,63)$ & & $1,25(1,13-1,39)$ & \\
\hline
\end{tabular}

ABEP: Associação Brasileira de Empresas de Pesquisa 17.

* Prevalência de ter consultado para os olhos nos últimos cinco anos;

** Análise ajustada para as variáveis dos níveis hierárquicos superiores do modelo;

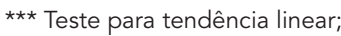

\# Teste de heterogeneidade;

\#\# Auto-relatado segundo a informação de um médico.

\section{Discussão}

Uma parte importante da população estudada, inclusive daqueles com 50 anos ou mais, não consultou para os olhos nos últimos cinco anos. Entre aqueles que consultaram, um número muito expressivo teve que pagar para consultar, e quase um quinto das pessoas consultou em ótica, enquanto o setor público atendeu apenas $17 \%$ dos entrevistados. O principal motivo de consulta foi não enxergar bem. Os principais motivos para não ter consultado, quando teve necessidade, foram falta de tempo e de dinheiro. Idade, escolaridade e nível econômico apresentaram associação direta com ter consultado nos últimos cinco anos. Ser mulher, referir ca- tarata, glaucoma ou usar correção ocular, bem como ter feito consulta por meio de convênio ou particular, também estiveram associados com o desfecho. No entanto, referir hipertensão arterial sistêmica, diabetes melito ou toxoplasmose não apresentaram associação com ter consultado nos últimos cinco anos.

Este estudo baseia-se numa amostra representativa da população da cidade. Sua validade interna é reforçada pelo baixo percentual de perdas e recusas. $\mathrm{O}$ estudo avaliou se a pessoa consultou nos últimos cinco anos para os olhos, entretanto não detalhou qual profissional realizou a consulta, nem em que medida os exames restringiram-se à avaliação do problema de refração ou implicaram exames mais completos 4. 
Além disso, a lembrança de utilização de serviço de saúde é pobre e declina com o tempo ${ }^{19}$. Porém, espera-se que, por se tratar de uma consulta especializada e, na maioria, paga, o recordatório seja melhor do que para consultas em geral. Outra limitação é que não foi medido o papel do nível de oferta de serviços de saúde na determinação de acesso e utilização de serviços.

O número de pessoas que não consultaram para os olhos nos últimos cinco anos ou nunca consultaram é preocupante, especialmente no grupo de 50 anos ou mais, uma vez que não atende a recomendação de uma periodicidade de cinco anos para a população em geral, nem a de dois anos para aqueles com morbidades crônicas com repercussão ocular 4 .

As prevalências de utilização encontradas foram semelhantes à de um estudo no Irã, que apontou $67,5 \%$ de utilização de serviços de saúde ocular nos últimos cinco anos, entre a população acima de 18 anos 6 . Por outro lado, foi inferior à encontrada em países desenvolvidos, como a Austrália, onde cerca de $80 \%$ dos indivíduos com 40 anos ou mais utilizaram serviços de saúde ocular nos últimos cinco anos 4,8, e $62 \%$ das pessoas de 49 anos ou mais o fizeram nos últimos dois anos 7. Foi inferior, também, à prevalência encontrada nos Estados Unidos, onde 53\% das pessoas com 55 anos ou mais de idade utilizaram serviços de saúde ocular no último ano 11 .

A avaliação das necessidades em saúde através de morbidade referida gera uma estimativa da prevalência de grande relevância, diante da inexistência de estudos nacionais populacionais sobre morbidades oculares. Isso é reforçado pela consistência, com a literatura, das prevalências de diabetes melito, toxoplasmose, glaucoma, catarata e uso de correção, tanto na população geral, quanto nos de 50 anos ou mais $1,20,21,22,23,24,25,26,27,28$. No caso da hipertensão arterial sistêmica, o presente estudo encontrou prevalência maior do que a encontrada por outro estudo realizado na mesma cidade. Entretanto, este tinha distribuição etária e critério para definir hipertensão arterial sistêmica diferentes 29 .

Com relação à forma de financiamento da última consulta para os olhos, observa-se que o setor público apresentou uma prevalência extremamente baixa (17\%), se comparado a consultas médicas em geral, caso em que o sistema público respondeu por $48 \%$ destas 30 . A participação do sistema público no que se refere à atenção à saúde ocular é ainda menor do que aquela voltada à atenção odontológica (mais de 20\%); nesta, a atuação do SUS é muito mais baixa do que na atenção médica 31 .

A baixa oferta de consultas no setor público se reflete no principal motivo para ter deixado de consultar quando sentiu necessidade, que foi falta de dinheiro. Os dois outros motivos mais prevalentes, falta de tempo e descaso, também podem estar relacionados à dificuldade de acesso ${ }^{32}$. Nesse caso, a pessoa se responsabiliza por não ter tentado conseguir uma consulta em situação de grande dificuldade, como as longas filas, os horários específicos para marcação de consultas, muitas vezes incompatíveis com suas possibilidades, a espera para consultar, a falta de dinheiro para transporte e o risco de investir grande esforço para tentar agendar e não conseguir. Em um estudo indiano, as principais barreiras enfrentadas pelas pessoas com 40 anos ou mais ao acesso a serviços de saúde ocular também foram falta de dinheiro $(78,2 \%)$ e falta de tempo $(70 \%) 13$.

O setor privado, portanto, tem um papel preponderante na atenção à saúde ocular, o que justifica a rapidez do atendimento (mais da metade em menos de 24 horas). Chama também atenção $\mathrm{o}$ alto percentual de consultas em óticas. Esta parece ser uma estratégia da população que tem dificuldade para pagar a consulta ou que não tem convênio para resolver, de forma rápida, seu problema de acuidade visual. Em muitos casos há, inclusive, venda "casada" de consulta e óculos, o que implica um problema ético.

Apesar da importância do setor privado, as consultas voltadas para a prevenção de morbidades oculares parecem restritas, uma vez que o principal motivo de consulta é não enxergar bem (mais de 70\%). Em dois estudos de base populacional, as dificuldades visuais também se apresentaram como o principal motivo para consultar $(45,8 \%$ e $88,6 \%)$ entre aqueles com 40 anos ou mais 13,14 .

Ao se examinarem os fatores associados com consulta para os olhos nos últimos cinco anos, observou-se que as mulheres consultaram mais do que os homens, o que está de acordo com outros estudos em que o risco de consultar foi 1,27 e 1,78 para mulheres 4,6,8,12. Segundo Sawyer et al. 33 , esse diferencial se explica, em parte, por um interesse maior do gênero feminino pela sua condição de saúde e por uma percepção e atitude diferentes em face das necessidades em saúde. A associação direta de idade com ter consultado para os olhos nos últimos cinco anos concorda com outros estudos 4,6,10,13,14 e está relacionada à prevalência mais alta dos erros refrativos, como a presbiopia, e das morbidades crônicas oculares e sistêmicas 1,3, especialmente entre aqueles acima dos 50 anos.

Indivíduos de cor branca consultaram mais do que os negros nos últimos cinco anos, concordando com estudos americanos 10,12. Por outro lado, discorda de outro estudo, entre mulheres, 
que apontou para o fato de que as negras consultaram mais do que as brancas 34 . Espera-se que os negros apresentem maiores necessidades em saúde ocular, porque a hipertensão arterial sistêmica e o glaucoma são mais prevalentes neles do que em brancos 29,34. A menor utilização dos serviços de saúde pelos negros pode estar relacionada ao menor conhecimento sobre saúde; à menor valorização das necessidades em saúde, à discriminação racial ou mesmo a um efeito de confusão residual de nível econômico ${ }^{34}$. Ademais, considerando que uma parte importante da atenção é realizada por óticas, os negros poderiam ter mais dificuldade para obter crédito.

A associação direta entre escolaridade e a utilização de serviços de saúde ocular também foi observada por outros estudos 6,7,12,13. O mesmo se deu entre nível econômico e o desfecho, estando de acordo com um estudo australiano que mostrou que pessoas com status sócio-econômico mais alto consultaram $30 \%$ mais 7 .

O melhor nível econômico e o maior conhecimento levam a uma identificação mais apurada das necessidades em saúde, a um comportamento de busca por serviços de saúde mais adequado e mais efetivo para resolver as necessidades identificadas e a uma inserção em redes sociais que facilitam o acesso aos serviços de saúde 6,35. O nível econômico mais alto possibilita arcar com custos relativos a transporte, cuidados com familiares, poder sair de casa, e outros, facilitando o acesso a todos os tipos de serviço de saúde, inclusive público. Isso é importante no contexto desse estudo, no qual o setor privado teve papel preponderante 36 .

A medida auto-referida de diabetes melito, hipertensão arterial sistêmica, toxoplasmose, catarata, glaucoma e uso de correção é adequada para examinar a associação entre as necessidades de saúde e ter consultado nos últimos cinco anos, uma vez que o fato de pensar ser portador da doença já deveria desencadear maior utilização de serviço de saúde ocular.

Diagnóstico médico de diabetes melito, hipertensão arterial sistêmica e toxoplasmose não estiveram associados com consulta nos últimos cinco anos. Esse achado está em desacordo com estudos em países desenvolvidos, onde os diabéticos têm uma freqüência entre $70 \%$ e $168 \%$ maior de consultas 8,10,11. A avaliação periódica da saúde ocular é de extrema importância para prevenção de cegueira e para diagnóstico precoce e tratamento da retinopatia diabética, que apresenta uma prevalência de cerca de $20 \%$ entre os diabéticos 37,38. A retinopatia diabética é responsável por cerca de $5 \%$ dos 37 milhões de casos de cegueira causada por doenças oculares no mundo 1 .
Em relação à hipertensão arterial sistêmica, o papel da avaliação periódica da saúde ocular não está claramente estabelecido, mas parece contribuir para a avaliação da gravidade do problema e para a adequação do tratamento ${ }^{39}$. A avaliação periódica é ainda importante não só para o diagnóstico precoce de toxoplasmose e suas recidivas, minimizando as lesões cicatriciais, como também para a pesquisa do papel da toxoplasmose adquirida no desenvolvimento da toxoplasmose ocular, particularmente no Rio Grande do Sul, onde a prevalência de sorologia positiva e a retinocoroidite toxoplásmica é bastante elevada em algumas regiões 40,41.

Assim, o fato de os portadores dessas morbidades não utilizarem mais a atenção à saúde ocular pode estar relacionado ao desconhecimento do impacto das morbidades sistêmicas sobre os olhos, à dificuldade de acesso aos serviços de saúde ou a dificuldades da atenção básica para encaminhar para outros níveis de atenção. Apesar de a diabetes melito e a toxoplasmose apresentarem baixas prevalências (menor do que $10 \%)$, considerou-se que o poder estatístico do estudo esteve adequado, especialmente porque se esperavam riscos relativos grandes.

Concordando com a literatura, o auto-relato de catarata esteve associado com a utilização de serviços de saúde ocular nos últimos cinco anos 7 . Dentre as patologias oculares, esta foi a morbidade mais freqüentemente relacionada com a dificuldade para enxergar ${ }^{42}$. A OMS estima que quase 18 milhões de pessoas, em todo o mundo, estão cegas bilateralmente devido à catarata 1; na América Latina, a doença é a principal causa de cegueira bilateral 43. A catarata não é um problema prevenível, entretanto a intervenção cirúrgica pode evitar a baixa visão e a cegueira. Assim, o manejo da morbidade requer retaguarda de outros níveis de atenção para realização do tratamento e do acompanhamento pós-cirúrgico.

Ao contrário da catarata, o glaucoma resulta em danos visuais irreversíveis, especialmente porque metade dos portadores da doença não sabe de sua condição ${ }^{44}$. Além da dificuldade de acesso à consulta, o acompanhamento do glaucomatoso sofre descontinuidade, pela dificuldade de acesso a exames complementares complexos, como é o caso da campimetria computadorizada. A realização de consultas periódicas para os olhos, especialmente acima dos 50 anos, possibilita o diagnóstico precoce e o monitoramento do progresso desta morbidade, fornecendo informações importantes para a adequação do tratamento ${ }^{4}$. Segundo a OMS, 4,5 milhões de pessoas, em todo o mundo, estão cegas devido ao glaucoma e, aproximadamente, 80 milhões de indivíduos terão a doença no ano de 20201. 
O uso de correção ocular foi o fator que se apresentou mais fortemente associado com consultar para os olhos nos últimos cinco anos, estando de acordo com a literatura11. Essa necessidade em saúde realmente parece desencadear a utilização de serviço de saúde ocular de forma próxima ao recomendado 4 . Todavia, em um percentual importante das vezes, a atenção ao problema refrativo é feita através de óticas, de forma que problemas oftalmológicos associados não são avaliados.

Consultar no setor privado esteve positivamente associado com consultar nos últimos cinco anos, o que concorda com estudo norteamericano que apontou três vezes mais consultas entre quem buscou o setor público e privado do que entre os que buscaram, exclusivamente, o setor público 11. Segundo outro trabalho, a probabilidade de utilizar um serviço de saúde ocular em um ano é maior entre norte-americanos que possuem convênio ou pagam por suas consultas do que entre aqueles exclusivamente dependentes do setor público 45 . Esse achado é plausível, porque o setor privado tem maior oferta de serviços, facilitando o acesso.

O presente estudo mostra que a prevalência de consultas nos últimos cinco anos foi aquém do recomendado e que a atenção foi prestada principalmente pelo setor privado, por oftalmologistas ou em óticas, concentrando o cuidado nos problemas de refração. Desse modo, torna-se necessário aumentar a participação do setor público, integrando a saúde ocular a todos os níveis de atenção ${ }^{1}$, além de incluir profissionais, na rede básica, que possam ampliar o rastreamento da acuidade visual e realizar tonometria para fazer triagem de glaucoma.

Em muitos países, o optometrista realiza cuidados em saúde ocular, particularmente a avaliação refrativa e a adequação da correção ocular, parecendo ser o profissional indicado para desempenhar ações de saúde dessa complexidade. No Brasil. tal alternativa tem encontrado grande resistência, mas poderia ser uma opção para enfrentar o problema visual mais freqüente na população: os erros de refração.

É importante, também, contar com profissionais direcionados para a atenção à saúde ocular nos Núcleos de Apoio à Saúde da Família, atendendo, prioritariamente, a população portadora de doenças oculares e de doenças sistêmicas com repercussão ocular. Essas ações devem ser complementadas com a ampliação do acesso a medicações, armações e lentes, exames complementares e procedimentos cirúrgicos. A partir dessas avaliações, será possível organizar a referência para os ambulatórios de oftalmologia e para os procedimentos a serem realizados em outros níveis de atenção, como o cirúrgico.

\section{Resumo}

Este estudo transversal de base populacional investigou a prevalência de utilização de serviços de saúde ocular e sua associação com fatores sócio-demográficos, necessidades em saúde e forma de financiamento da consulta. Avaliaram-se 2.960 indivíduos de 20 anos ou mais. Nos últimos cinco anos, $46 \%$ dos entrevistados e $30 \%$ daqueles com 50 anos ou mais não consultaram para os olhos. Dos que consultaram, 18\% foram em óticas, e apenas $17 \%$ foram no setor público. $O$ principal motivo foi não enxergar bem (69,5\%). Falta de dinheiro (29\%) e de tempo (24,6\%) foram os principais motivos para ter deixado de consultar. Idade, escolaridade e nível econômico estiveram diretamente associados com ter consultado nos últimos cinco anos.
Ser mulher, ter catarata, glaucoma e usar correção, bem como consultar no setor privado também estiveram positivamente associados com o desfecho. É preciso não só aumentar a participação do setor público, integrando a saúde ocular a todos os níveis de atenção, ampliando a participação de outros profissionais de saúde, como também intensificar o rastreamento de problemas oculares e a sua prevenção.

Serviços Oftalmológicos; Saúde Ocular; Setor Público 


\section{Colaboradores}

V. D. Castagno atuou na concepção do projeto, análise de dados, interpretação dos resultados, redação do artigo, e aprovou a versão final a ser publicada. A. G. Fassa participou da concepção do projeto, análise de dados, interpretação dos resultados, redação do artigo, e aprovou a versão final a ser publicada. M. C. Silva atuou na concepção do projeto, revisão crítica do artigo, e aprovou a versão final a ser publicada. M. L. V. Carret contribuiu com a análise e interpretação dos dados, revisão crítica do artigo, e aprovou a versão final a ser publicada.

\section{Referências}

1. World Health Organization/International Agency for the Prevention of Blindness. Data Vision 2020: the right to sight. Global initiative for the elimination of avoidable blindness. Action plan 2006-2011. Geneva: World Health Organization/International Agency for the Prevention of Blindness; 2007.

2. Instituto Brasileiro de Geografia e Estatística. Pesquisa nacional por amostragem de domicílios, 2000. Rio de Janeiro: Instituto Brasileiro de Geografia e Estatística; 2001.

3. Taylor HR, Vu HT, McCarty CA, Keeffe JE. The need for routine eye examinations. Invest Ophthalmol Vis Sci 2004; 45:2539-42.

4. Bylsma GW, Le A, Mukesh BN, Taylor HR, McCarty CA. Utilization of eye care services by Victorians likely to benefit from eye care. Clin Experiment Ophthalmol 2004; 32:573-7.

5. American Optometric Association. Recommended eye examination frequency for pediatric patients and adults. http://www.aoa.org/x5502.xml (acessado em 18/Nov/2007).

6. Fotouhi A, Hashemi H, Mohammad K. Eye care utilization patterns in Tehran population: a population based cross-sectional study. BMC Ophthalmol 2006; 6:4.

7. Wang JJ, Mitchell P, Smith W. Use of eye care services by older Australians: the Blue Mountains Eye Study. Aust N Z J Ophthalmol 1999; 27:294-300.

\section{Agradecimentos}

Ao Conselho Nacional de Desenvolvimento Científico e Tecnológico (CNPq) através do Edital Universal (no. 480476/2007-1) e da bolsa de produtividade científica da pesquisadora Anaclaudia Gastal Fassa.
8. Keeffe JE, Weih LM, McCarty CA, Taylor HR. Utilisation of eye care services by urban and rural Australians. Br J Ophthalmol 2002; 86:24-7.

9. Laitinen A, Koskinen S, Rudanko SL, Martelin T, Laatikainen L, Aromaa A. Use of eye care services and need for assistance in the visually impaired. Optom Vis Sci 2008; 85:341-9.

10. Zhang X, Saaddine JB, Lee PP, Grabowski DC, Kanjilal S, Duenas MR, et al. Eye care in the United States: do we deliver to high-risk people who can benefit most from it? Arch Ophthalmol 2007; 125:411-8

11. Puent BD, Klein BE, Klein R, Cruickshanks KJ, Nondahl DM. Factors related to vision care in an older adult cohort. Optom Vis Sci 2005; 82:612-6.

12. Orr P, Barron Y, Schein OD, Rubin GS, West SK. Eye care utilization by older Americans: the SEE Project. Salisbury Eye Evaluation. Ophthalmology 1999; 106:904-9.

13. Nirmalan PK, Katz J, Robin AL, Krishnadas R, Ramakrishnan R, Thulasiraj RD, et al. Utilisation of eye care services in rural south India: the Aravind Comprehensive Eye Survey. Br J Ophthalmol 2004; 88:1237-41.

14. Palagyi A, Ramke J, du Toit R, Brian G. Eye care in Timor-Leste: a population-based study of utilization and barriers. Clin Experiment Ophthalmol 2008; 36:47-53. 
15. Conselho Brasileiro de Oftalmologia. Censo 2001: distribuição dos oftalmologistas por estado. São Paulo: Conselho Brasileiro de Oftalmologia; 2001.

16. Departamento de Informática do SUS, Ministério da Saúde. Caderno de informações de saúde. Brasília: Departamento de Informática do SUS, Ministério da Saúde; 2007.

17. Associação Brasileira de Empresas de Pesquisa. Critério de classificação econômica Brasil. São Paulo: Associação Brasileira de Empresas de Pesquisa; 2003.

18. Andersen RM. Revisiting the behavioral model and access to medical care: does it matter? J Health Soc Behav 1995; 36:1-10.

19. Evans E, Crawford B. Patient self reports in pharmacoeconomic studies. Their use and impact on study validity. Pharmacoeconomics 1999; 15: 241-56.

20. Malerbi D, Franco L. The Brazilian Cooperative Group on the Study of Diabetes Prevalence Multicenter: study of the prevalence of diabetes mellitus and impaired glucose tolerance in the urban Brazilian population aged 30-69 yr. Diabetes Care 1992; 15:1509-16.

21. Orefice F. Uveíte: clínica \& cirurgia: texto \& atlas. Rio de Janeiro: Cultura Médica; 2000.

22. Glasner P, Silveira C, Kruszon-Moran D, Martins M, Burnier Junior M, Silveira S, et al. An unusually high prevalence of ocular toxoplasmosis in southern Brazil. Am J Ophthalmol 1992; 114:136-44.

23. Varma R, Ying-Lai M, Francis BA, Nguyen BB, Deneen J, Wilson MR, et al. Prevalence of open-angle glaucoma and ocular hypertension in Latinos: the Los Angeles Latino Eye Study. Ophthalmology 2004; 111:1439-48.

24. Ramakrishnan R, Nirmalan PK, Krishnadas R, Thulasiraj RD, Tielsch JM, Katz J, et al. Glaucoma in a rural population of southern India: the Aravind Comprehensive Eye Survey. Ophthalmology 2003; 110:1484-90.

25. Khandekar R, Jaffer MA, Al Raisi A, Zutshi R, Mahabaleshwar M, Shah R, et al. Oman Eye Study 2005: prevalence and determinants of glaucoma. East Mediterr Health J 2008; 14:1349-59.

26. Acosta R, Hoffmeister L, Román R, Comas M, Castilla M, Castells X. Revisión sistemática de estudios poblacionales de prevalencia de catarata. Arch Soc Esp Oftalmol 2006; 81:509-16.

27. Bassett KL, Noertjojo K, Liu L, Wang FS, Tenzing C, Wilkie A, et al. Cataract surgical coverage and outcome in the Tibet Autonomous Region of China. $\mathrm{Br}$ J Ophthalmol 2005; 89:5-9.

28. Duarte WR, Barros AJD, Dias-da-Costa JS, Cattan JM. Prevalência de deficiência visual de perto e fatores associados: um estudo de base populacional. Cad Saúde Pública 2003; 19:551-9.

29. Piccini RX, Victora CG. Hipertensão arterial sistêmica em área urbana no sul do Brasil: prevalência e fatores de risco. Rev Saúde Pública 1994; 28:261-7.

30. Rosa Filho LA, Fassa AG, Paniz VMV. Fatores associados à continuidade interpessoal na atenção à saúde: estudo de base populacional. Cad Saúde Pública 2008; 24:915-25.
31. Barros A, Bertoldi A. Desigualdades na utilização e no acesso a serviços odontológicos: uma avaliação em nível nacional. Ciênc Saúde Coletiva 2002; 7:709-17.

32. Donoghue M. People who don't use eye services: 'making the invisible visible'. Community Eye Health 1999; 12:36-8.

33. Sawyer DO, Leite IC, Alexandrino R. Perfis de utilização de serviços de saúde no Brasil. Ciênc Saúde Coletiva 2002; 7:757-76.

34. Schaumberg DA, Christen WG, Glynn RJ, Buring JE. Demographic predictors of eye care utilization among women. Med Care 2000; 38:638-46.

35. Pinheiro RS, Travassos C. Estudo da desigualdade na utilização de serviços de saúde por idosos em três regiões da cidade do Rio de Janeiro. Cad Saúde Pública 1999; 15:487-96.

36. Mendoza-Sassi R, Béria JU. Utilización de los servicios de salud: una revisión sistemática sobre los factores relacionados. Cad Saúde Pública 2001; 17:819-32.

37. McCarty C, Lloyd-Smith C, Lee S, Livingston PM, Stanislavsky YL, Taylor HR. Use of eye care services by people with diabetes: the Melbourne visual Impairment project. Br J Ophthalmol 1998; 82:410-4.

38. Raman R, Rani PK, Reddi Rachepalle S, Gnanamoorthy P, Uthra S, Kumaramanickavel G, et al. Prevalence of diabetic retinopathy in India: Sankara Nethralaya Diabetic Retinopathy Epidemiology and Molecular Genetics Study report 2. Ophthalmology 2009; 116:311-8.

39. Chobanian A, Bakris G, Black H, Cushman WC, Green L, Izzo J. The seventh report of the joint national commiottee on prevention, detection, evaluation and treatment of high blood pressure: the JNC 7 report. JAMA 2003; 289:2560-72.

40. Silveira C, Belfort Jr. R, Muccioli C, Abreu MT, Martins MC, Victora C, et al. A follow-up study of Toxoplasma gondii infection in southern Brazil. Am J Ophthalmol 2001; 131:351-4.

41. Jones J, Muccioli C, Belfort Junior R, Holland GN, Roberts J, Silveira S. Recently acquired Toxoplasma gondii infection, Brazil. Emerg Infect Dis 2006; 12:582-7.

42. Nirmalan PK, Thulasiraj RD, Maneksha V, Rahmathullah R, Ramakrishnan R, Padmavathi A, et al. A population based eye survey of older adults in Tirunelveli district of south India: blindness, cataract surgery, and visual outcomes. Br J Ophthalmol 2002; 86:505-12.

43. Limburg H, Barria von-Bischhoffshausen $F$, Gomez P, Silva JC, Foster A. Review of recent surveys on blindness and visual impairment in Latin America. Br J Ophthalmol 2008; 92:315-9.

44. Wensor M, McCarty C, Stanislavisky YL, Livingston PM, Taylor HR. The prevalence of glaucoma in the Melbourne Visual Impairment Project. Ophthalmology 1998; 105:733-9.

45. Zhang X, Lee PP, Thompson TJ, Sharma S, Barker L, Geiss LS, et al. Health insurance coverage and use of eye care services. Arch Ophthalmol 2008; 126:1121-6.

Recebido em 12/Dez/2008

Versão final reapresentada em 05/Mar/2009

Aprovado em 29/Abr/2009 